PS1 - 186

doi:10.1017/cjn.2016.362

\section{Needs Assessment for a Pediatric Proton Therapy Program in Canada}

S. Patel ${ }^{1}$, J. A. Whitlock ${ }^{2}$, I.A. Olivotto ${ }^{3}$, M. Parliament ${ }^{l}, N$.

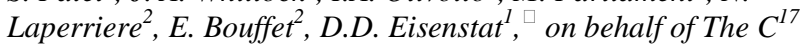
Council

1University of Alberta, Edmonton, $A B$

${ }^{2}$ University of Toronto, Toronto, $O N$

${ }^{3}$ University of Calgary, Calgary, $A B$

samir.patel2@albertahealthservices.ca

Proton therapy enables normal tissue sparing for curative-intent treatment of children with cancer who require radiation therapy. In the USA and elsewhere, proton therapy is being rapidly adopted, and many new proton centres are being established. Without a proton centre in Canada, children and their families must travel abroad for treatment at high cost and has raised the question whether a Canadian proton therapy facility is needed. METHODS: Canadian Pediatric Oncology centres were surveyed to assess current and future clinical practices. Needs were modeled by screening the Alberta Cancer Registry, ascertaining the number of children eligible for proton RT and comparing to the number who actually received this therapy. RESULTS: Most centres (63\%) referred children, and 49 children were referred abroad between 2008 and 2013. Referrals were estimated to increase to 36 cases per annum across Canada. Most respondents (75\%) supported that proton therapy will reduce late effects in most or selected cases compared to photon therapy. The registry search revealed 37,170 patients irradiated of which 379 children $(1.0 \%)$ were potentially eligible for proton therapy, accounting for $15.9 \%$ of the new cases of childhood cancers diagnosed in Alberta over the interval. CONCLUSIONS: A strong perceived need for a pediatric proton therapy in Canada was identified. Proton therapy utilization was lower than modeled needs. Future referrals are anticipated to increase, with annual estimated cost of approximately $\$ 60$ million spent outside of Canada that could be invested within the Canadian health care economy. These issues are worthy of further national discussion.

PS1 - 218

doi:10.1017/cjn.2016.363

\section{Characterization of Ganglioglioma as a Neurodevelopmental Disorder: A Cast of Arrested Development?}

\section{Q. Jiang ${ }^{I}$, J. Zagozewski, P. Nozza, B.Wilson, F. Van Landeghem, D.D Eisenstat \\ ${ }^{1}$ University of Alberta, Edmonton, $A B$ \\ eisensta@ualberta.ca}

Gangliogliomas (GG) are low grade neuroepithelial tumours of the central nervous system (CNS) comprised of neoplastic glial and neuronal cells. There are no animal models or cell lines to study. Microarray data of a panel of low grade gliomas including GG revealed overexpression of the DLX2 homeobox gene required for tangential interneuronal migration and differentiation in the embryonic forebrain. We hypothesized that DLX2 regulates neural versus glial cell fate decisions in CNS progenitors and that GG are arrested in development. METHODS: DLX2 expression was examined along with glial fibrillary acidic protein (GFAP; glial marker) and synaptophysin and/or NeuN (neuronal markers) expression in a cohort of GG tumours using immunohistochemistry and dual immunofluorescence labelling of formalin fixed paraffin embedded (FFPE) tissue sections. BRAF mutational status was also assessed. RESULTS: In our discovery cohort (Genoa), 10/30 samples $(33 \%)$ expressed DLX2. In our validation cohort (Edmonton), 22/36 (61\%) expressed nuclear DLX2 and 12/22 DLX2+ cases had BRAF mutations (55\%; 11 V600E, 1 V600G). $12 / 18$ cases with BRAF mutations were DLX2+ (67\%). One heavily pre-treated child with progressive cervicomedullary GG has had a very good partial response to BRAF inhibitor therapy. All 22 DLX2+ tumours co-expressed GFAP (100\%) and 21/22 (95\%) also expressed synaptophysin or NeuN. CONCLUSIONS: Our results support GG as neurodevelopmental tumours arising from bipotential CNS progenitors that are arrested at the neuralglial cell fate "decision" point. Biological or differentiation-based treatments could be considered +/- BRAF inhibitors for those GG with/without the V600E mutation, respectively.

\section{SCIENTIFIC POSTER SESSION II 10 JUNE $2016 \sim 1730-1830$}

\section{GLIOMA BASIC SCIENCE}

PS2 - 153

doi:10.1017/cjn.2016.364

\section{Dianhydrogalactitol (VAL-083) Treatment Causes Irreparable DNA Double-Strand Breaks, S/G2 Phase Cell-Cycle Arrest and Cell Death in Cancer Cells}

\section{B. Zhai, A. Steino, J. Bacha, D. Brown, M. Daugaard asteino@delmarpharma.com}

Dianhydrogalactitol (VAL-083) is a unique bi-functional alkylating agent causing N7-guanine-methylation and inter-strand DNA crosslinks. VAL-083 readily crosses the blood-brain barrier, accumulates in brain tumor tissue and has shown activity in prior NCI-sponsored clinical trials against various cancers, including glioblastoma (GBM) and medulloblastoma. VAL-083 is also active against GBM cancer stem cells and acts as a radiosensitizer independent of O6-methylguanine-DNA methyltransferase activity (in contrast to e.g. temozolomide and BCNU). Here we report new insights into VAL-083 mechanism of action by showing that VAL083 induces irreversible cell-cycle arrest and cell death caused by replication-dependent DNA damage. In lung (H2122, H1792, H23, A549) and prostate (PC3, LNCaP) cancer cell lines VAL-083 treatment caused irreversible S/G2 cell-cycle arrest and cell death (IC50 range 3.06-25.7 $\mu \mathrm{M}$ ). VAL-083 pulse-treatment led to persistent phosphorylation of DNA double-strand breaks (DSB) sensors ATM, single-strand DNA-binding Replication Protein A (RPA32), and histone variant H2A.X, suggesting persistent DNA lesions. After 10 months in culture with increasing VAL-083 concentrations, H1792 and LNCaP cells survive at concentrations up to $9.4 \mu \mathrm{M}$ and $7.4 \mu \mathrm{M}$, respectively, suggesting that efficient resistance mechanisms are not easily acquired by the cancer cells. Taken together with previous results showing that VAL-083 